\title{
The Benefits of Calorie Restriction and Calorie Restriction Mimetics as Related to the Eye
}

\author{
T.S. Anekonda*
}

Department of Neurology, Oregon Health and Science University, Portland, OR 97239-3098, USA

\begin{abstract}
The effects of calorie restriction without malnutrition seem to possess many beneficial effects in numerous disease states. Recently, studies related to calorie restriction mimetics that biochemically mimic the effects of calorie restriction are also becoming increasingly popular. Both calorie restriction and calorie restriction mimetics trigger an adaptive response reminiscent of mild-stress or low-dose toxic response, which is frequently referred to as hormesis in the toxicology literature. Although some benefits of calorie restriction and calorie restriction mimetics have been studied, the role of hormesis-related pathways in the eye has not been given a special attention. This review will present the current literature on calorie restriction and calorie restriction mimetics as related to most prominent eye diseases and provide insights on the therapeutic role of hormesis in eye diseases.
\end{abstract}

\section{INTRODUCTION}

The health benefits of calorie restriction $(\mathrm{CR})$ have been known to the scientists for decades. In the recent literature, $\mathrm{CR}$ or dietary restriction has been generally defined as consumption of nutritious diet that is $40 \%$ less in calories compared to ad libitum diet. In addition to providing protection against numerous deadly diseases such as cancer, neurological disorder, and obesity, CR is the only reliable treatment that extends life span or causes healthy aging consistently in a multitude of organisms ranging from bacteria to monkeys [1-4]. The extent to which CR extends human life span, however, is still largely unknown. The most frequently touted effect of CR has been its influence on creating a mild stress in the organism and a typical up-regulation of adaptive mechanisms involving stress proteins accompanied by elevated defense or survival molecules [5-7]. This response is similar to the expected hormetic response [8]. According to the theory of "hormesis," toxins and pollutants generally show biphasic dose response, where a low dose of toxin triggers a positive, adaptive stress response, which may help an organism sustain much higher levels of toxins which otherwise cause harmful effects [7-12].

Although CR can benefit human health and extend longevity, its success depends on determined change in human behavior-willingness to mildly starve! Ironically, the current calorie consumption trend in the United States is just the opposite, with weight gain and obesity expected to increase in the coming decades [13]. Realizing that it is difficult to influence public calorie consumption patterns, scientists have been contemplating alternative ways to accrue the benefits of CR without actually suggesting restriction of calorie intake. Such a strategy is made possible because a large number of plant-derived chemicals or phytochemicals can mimic the effect of CR. These phytochemicals are referred to as CR mimetics (CRMs) [14, 15]. CRMs at low

*Address correspondence to this author at the Department of Neurology, Oregon Health and Science University, Portland, OR 97239-3098, USA; Tel: 503-494-5023; E-mail: anekondt@ohsu.edu doses function like toxicants and trigger the adaptive response in the organism [8-10, 12]. Fruits, nuts, vegetables and herbs together offer an estimated 4000 different kinds of flavonoids [16-18] that may have been evolved to fight diseases and insect attack [14], but at proper dosage levels may be extremely beneficial to human health. The popularity of herbals can be attributed to the public belief that herbs are naturally safer than synthetic drugs [19]. Experts believe that one in three Americans uses herbal supplements, with the consumption level much greater among women [20, 21], patients undergoing surgery [22], and the elderly. Because herbals seem to embody both medical and marketing benefits, scientists are excited about their prospects as suitable alternatives to the $\mathrm{CR}$.

Both CR and CRMs affect a common pool of biochemical pathways that are implicated in organism's survival and longevity. Specifically, the following two biochemical pathways and associated proteins have shown much promise in the last decade: (1) Sirtuin pathway [5, 6, 23-25], and (2) Kelch-like ECH-associated protein 1 (Keap1)/nuclear factor erythroid 2-related factor 2 (Nrf2)/antioxidant response element (ARE) pathway, simply referred to as the Keap1/Nrf2/ARE pathway [26]. While anti-aging sirtuin pathway is implicated in an organism's longevity [25], ARE pathway is known to trigger the upregulation of cytoprotective genes essential for cell survival [26]. Emphasis in the eye literature addressing these specific pathways is limited and only currently emerging literature seems to provide some initial insights into the roles of these pathways in the eye. This review will focus on the benefits of CR and CRMs as related to the eye, particularly recognizing the potential hormetic pathways essential for survival and longevity.

\section{DISEASE AND ANATOMY OF THE EYE}

In epidemiological and nutritional supplement studies, traditionally known antioxidants (vitamins A, C, E) and carotenoids (lycopene, lutein, zeaxanthin) appear to have yielded a varying, but inconsistent degree of effectiveness in minimizing the damage caused by age-related eye diseases 
such as cataract, age-related macular degeneration, and glaucoma [27-29]. A broad range of herbal compounds has also been found beneficial to delaying age-related eye diseases [27-29].

The human eye, frequently referred to as the window to the mind, suffers from many diseases that may ultimately cause blindness. According to 2000 US Census, nearly 1 million Americans older than 40 years of age were blind, with an additional 2.4 million suffering from low vision [30]. The leading causes of blindness and low vision included agerelated macular degeneration (ARMD), cataract, glaucoma, diabetic retinopathy, and other diseases [30]. Inflammation in the eye including uveitis causes about $10 \%$ of blindness in the United States. The blindness in the US is projected to increase $70 \%$ by 2020 , largely due to the aging population [30]. Because most debilitating eye pathologies are agerelated, the treatments that modulate aging pathology or agerelated biochemical pathways are expected to contribute significantly for developing suitable treatments for age-related diseases.

The diseases and pertinent treatments of the eye can be better understood by knowing the basic anatomy of the eye, so a brief description of the human eye anatomy will be presented below. The anterior portion of the eye constitutes cornea, anterior chamber, iris, and the anterior ciliary muscle. The eye lens divides the anterior chamber from the posterior segment, which includes the posterior ciliary body, vitreous fluid, retina that surrounds the vitreous fluid, choroid, and the outer sclera. The iris, ciliary body and choroid together form the middle pigmented layer of the eye called the uvea. The cross-section of different layers of the eye from retina through sclera show complex layers of cells that perform specialized functions. The retina is a thin layer of transparent nerve tissue with at least five distinct types of nerve cells: the outermost photoreceptor cells (rods and cones), horizontal cells, bipolar cells, amacrine cells, and ganglion cells. The photoreceptor cells receive the light and transmit it to the other nerve layers all the way down to the ganglion cell layer. The retinal ganglion cells forming the optic nerve transmit the light signals to the brain. Immediately adjacent to the outer photoreceptor segment is a single layer of cells called retinal pigment epithelial cells (RPE), which among other things perform crucial phagocytosis function to recycle the debris that was created by the dead photoreceptor cells. The nourishment and oxygen supply to the retina is provided by the choroid, which in turn has four layers: the inner Bruch's membrane, choriocapillaris with small capillary vessels, a layer with medium-sized blood vessels (Sattler's layer), and the outermost layer with large vessels (Haller's layer). The outer most protective layer of the eye is called sclera.

\section{CALORIE RESTRICTION}

CR studies elsewhere in the body have re-emerged with a renewed interest in the recent years, especially in research related to cancer, obesity, and the brain. Eye research, however, appears to have fallen short in identifying the emerging beneficial molecular pathways of CR to sight-related diseases. Only a limited literature is available on the beneficial effects of CR against cataract, ARMD, and glaucoma, and this evidence is discussed below.

\section{Benefits of CR in Cataract}

Several animal studies have shown the beneficial effects of CR diet against cataract, the development of clouding in the crystalline lens of the eye leading to a progressive increase in the lens opacity associated with the loss of transparency [31,32]. Calorie restriction (at 60-79\% of ad libitum, AL, intake) in Emory mice, delayed the onset, formation, progression, and accumulation of cataract, besides extending the median life span of the animals by $40 \%$ [33-36]. Interestingly, the delayed accumulation of cataract did not correlate with the level of antioxidant enzymes [37]. In B6D2F1 mice (C57BL/6 x DBA/2), CR diet attenuated the decline of proliferative capacity of lens epithelial cells (LE) in older mice [38], and in old, CR mice LE cells were more resistant to $\mathrm{H}_{2} \mathrm{O}_{2}$-induced cell damage than LE cells in old, AL mice [39]. Of the two rat strains (Brown Norway \& Fischer 344), and three mouse strains (C57BL/6, (C57BL/6 x DBA/2)F1, (C57BL/6 x C3H)F1), CR diet extended life span in all strains, and delayed the first appearance and subsequent severity of cataract in four dark-eyed strains, but not in albino, Fischer 344 rats [40]. Further, in Brown Norway rats, $\mathrm{CR}$ diet attenuated age-related shortening of telomeres in LE cells [41]; retarded age-related degeneration of lens by reducing oxidative stress in the lens [42]; and, prevented agerelated decline in glycolytic enzymes, molecular chaperones, and $\alpha \mathrm{B}$-crystallin, a lens protein [43]. In Wistar rats, 50\% food restriction and $75 \%$ protein restriction lowered $\beta$-and $\gamma$ crystallin aggregation and the chaperone activity of $\alpha$ crystallin was improved by $50 \%$ vitamin restriction [44]. These studies clearly suggest that CR diet enhances an animal's longevity irrespective of the pigmentation, and protection against cataract occurs only in pigmented animals, but not in albinos. This anomaly in albino rodents may be related to their greater susceptibility to light-induced damage than the normal brown rodents.

\section{Benefits of CR in Age-Related Macular Degeneration}

ARMD is the leading cause of blindness in white Americans and it accounts for $54 \%$ of all blindness [30]. In ARMD, the increased degeneration of macula, a $5.5 \mathrm{~mm}$ area around fovea, occurs with aging and more severely after 60 years of age. One of the hallmarks of ARMD includes the extracellular deposition of misfolded and aggregated proteins, drusen bodies, beneath RPE and Bruch's membrane. Although age is the strongest risk factor for ARMD, several other systemic risk factors have also been identified: hypertension, smoking, and family history [45]. The degeneration and death of photoreceptor cells in the macula leads to the loss of central vision, then a progressive loss of peripheral vision. In most severe case, the in-growth of blood vessels from choroid rupture the Bruch's membrane and move into the sub-RPE and sub-retinal space leading to choroidal neovascularization [45]. Surprisingly, compared to CR studies in cataract, CR-related studies in ARMD are less conclusive. Given the fact that CR effects possess sirtuin as well as Keap1/Nrf2/ARE pathways and the prominent involvement of these pathways in the ARMD, the lack of CR-related studies as related to ARMD is astonishing. In Wistar rats, CR diet decreased the accumulation of lipofuscin in RPE cells [46]. Lipofuscin is an aggregate of complex material that accumulates within the lysosomes of RPE cells as a result of phagocytosis of degraded photoreceptor cells [47, 48]. The 
age-related accumulation of lipofuscin may occur at an accelerated rate and cause RPE dysfunction and rapid aging of retina $[47,48]$. In monkeys fed $70 \% \mathrm{CR}$ diet, no differences in drusen were detected between CR and AL groups, but the authors suggest that the sample size in this study was too small to conclude definitively [49]. In albino Fischer 344 rats, the deleterious effect of bright light was more pronounced in rats fed CR than in AL diet [50]. However, in Brown Norway rats, CR diet exhibited a neuroprotective effects in the aging retina [2], reduced age-related photoreceptor cell death, and the reduced decline of thiols, glutathione, ascorbic acid, and taurine in the retina [51]. Although not specifically substantiated by these limited number of available studies, CR appears to possess a neuroprotective effect in retina and a potential activation of protective Keap1/Nrf2/ARE pathway in ARMD.

\section{Benefits of CR in Glaucoma}

Increased intraocular pressure, caused by defects in the ocular drainage system, is the main risk factor for glaucoma [52-54]. Increased intraocular pressure can lead to the optic nerve damage and a subsequent loss of vision and blindness. In addition, age, diabetes, and genetic factors strongly influence the occurrence of glaucoma [55]. The most common form of glaucoma is a slowly developing, chronic form of primary open-angle glaucoma (POAG). In an animal study on POAG, when albino Fischer rats, albino Wistar rats, and Brown Norway rats were fed three days a week in a CR regimen, $\mathrm{CR}$ protected against the loss of retinal ganglion cells (RGCs) in both young and old animals and in both albino and pigmented rats [56]. CR diet also attenuated the loss of age-related RGCs in both albino BALB/cBy mice and albino Fischer rats [57], and resisted age-related morphological alterations in aqueous collecting channel in terms of the loss of lumen area of channel and loss of anteriorposterior width [58]. Further, in albino Fischer 344 rats, CR diet attenuated age-related as well as ischemia-induced RGC loss [59]. These studies strongly suggest CR has protective effects against the loss of RGCs irrespective of the pigment color of the animal. Because RGCs express SIRT1 and SIRT1 activation provides protection against optic neuritis primarily by protecting RGCs [60], it is possible that CRinduced upregulation of SIRT1 in RGCs can also provide protection against glaucoma.

\section{CALORIE RESTRICTION MIMETICS}

CR effects have been experimentally tested on a limited number of eye diseases such as, cataract, ARMD, and glaucoma, as discussed in the previous sections. Interestingly, CRMs seem to have been tested on a broader group of eye diseases including both age-related eye diseases and eye diseases that affect all ages. Although potentially many phytochemicals may qualify to serve as CRMs, resveratrol, sulforaphane, and curcumin appear to have been tested most commonly in the eye literature, so we will discuss these three herbals along with other compelling CRMs as related to the eye disease.

\section{Benefits of Resveratrol in Eye Diseases}

There is mounting evidence in the literature suggesting the beneficial effects of resveratrol consumption and red wine drinking. Despite the fat-rich diets consumed by the
French, an epidemiological finding, popularly known as "The French Paradox" has associated regular red wine consumption with a low incidence of cardiovascular disease [61]. A low incidence of vision loss among the elderly has also been linked to red wine drinking [27, 62]. Red wine and its biological source-the purple grape (Vitis vinifera), especially its skin and seeds-contain many polyphenols, such as flavonoids (quercetin, catechins, gallocatechin, procyanidin, prodelphidins), and resveratrol, a phytoalexin that is naturally synthesized in response to fungal attack [63-65]. In addition to its anti-aging properties, resveratrol is known to play a neuroprotective role in Huntington's disease [66], axotomy [67], Alzheimer's disease [68], brain ischemia [69], stroke [70] and epilepsy [71] by protecting brain cells from death. Recent studies also directly link the beneficial effects of resveratrol and SIRT1 activators with prevention of vision loss $[27,62,72,73]$ and activation of SIRT1 in the eye or eye cells $[60,74]$.

Resveratrol prevented sodium selenite-induced oxidative stress and cataract formation in an experimental cataract model in SD rats [73]. Acting as an antioxidant, resveratrol not only reduced the oxidative stress of the retinal pigment epithelium (RPE) cells, but also attenuated hyperproliferation of human RPE cells used as an in vitro model for ARMD [75]. In aging pigment epithelial cells, resveratrol quenched singlet oxygen and reduced A2E epoxidation, reducing the incidence of DNA damage and cell death [72]. Further, in human RPE cells, the expression of SIRT1 attenuated FOXO3 recruitment to the complement factor $\mathrm{H}$ $(\mathrm{CFH})$ regulatory region and reversed the $\mathrm{H}_{2} \mathrm{O}_{2}$-induced repression of $\mathrm{CFH}$ gene expression [76]. In optic neuritis and multiple sclerosis patients, axonal damage causes vision loss and neuronal dysfunction. Although the inflammation was not suppressed, modified resveratrol formulations (SRT647 and SRT501) prevented RGC loss and protected mice from neuronal damage in experimental autoimmune encephalomyelitis (EAE), an animal model for optic neuritis and multiple sclerosis [60]. In a 34-yr old man suffering from optic neuritis, administration of red wine $(0.3 \mathrm{dl})$ improved the visual acuity within 30 minutes of drinking wine, and provided temporary improvement in peripheral blood flow and visual function [77]. Among other symptoms, diabetic retinopathy is characterized by impaired ocular circulation and angiogenesis. In porcine retinal arterioles, an in vitro model for diabetic retinopathy, resveratrol $(1-50 \mu \mathrm{M})$ induced the endothelium-dependent dilation or relaxation of arterioles, and this dilation was mediated by the released nitric oxide (NO) via NO synthase (NOS) activation by the extracellular signal-regulated (ERK) pathway, and the subsequent activation of soluble guanylyl cyclase [78]. In a pressure-induced cultured retinal ganglion cells (RGC-5), an in vitro model for glaucoma, resveratrol $(20-40 \mu \mathrm{M})$ provided protection against increased oxidative stress and 4-hydroxy-2-nonenal (HNE) adducts and in C57LB/6 mice, the increased intraocular pressure $(10-60 \mathrm{Hg}$ ) dose-dependently increased the formation of $\mathrm{HNE}$ and the expression of protective protein heme oxygenase-1 (HO-1) in the mice retina [79]. The trabecular meshwork (TM) cells control the outflow of aqueous humor and thus intraocular pressure in glaucoma. In pig TM cells exposed to oxidative stress (40\% oxygen), resveratrol $(25 \mu \mathrm{M})$ treatment effectively prevented increased production of intracellular reactive oxygen species (iROS) and in- 
flammatory markers (interleukin (IL)1a, IL6, IL8; and endothelial leukocyte adhesion molecule 1, ELAM-1), and reduced the expression of the senescence markers sa-b-gal, lipofuscin, and the accumulation of carbonylated proteins [80]. In addition, resveratrol has exhibited anti-angiogenic effects in the eye. In C57B16/J mice implanted with vascular endothelial growth factor (VEGF) and fibroblast growth factor 2 (FGF-2), resveratrol inhibited corneal neovascularization [81], and in mice injected with tumor cells that cause uveal melanoma, resveratrol $(0.5-20 \mu \mathrm{M})$ inhibited both tumor size and volume through induction of mitochondriamediated and caspases-3 \& -9 mediated apoptosis the tumor cells [82]. Furthermore, resveratrol (50-100 $\mu \mathrm{M})$ suppressed the cell proliferation and increased mitochondria-mediated apoptosis in Y79 retinoblastoma cells [83]. In our recent study with E1A.NR3 retinal cells, Bax was upregulated in response to the antibody treatment, but resveratrol-induced SIRT1 activated Ku70 expression in the cytoplasm and this $\mathrm{Ku} 70$ appear to have suppressed the movement of Bax from cytoplasm to mitochondria and prevented antibody-induced apoptosis [74]. These studies clearly suggest broad therapeutic roles of resveratrol in the eye. Resveratrol seem to possess anti-oxidant, anti-apoptotic, anti-inflammatory, antiangiogenic, and an ability to induce both anti-aging sirtuin and Keap1/Nrf2/ARE pathways.

The previous studies have shown that CR and CRMs upregulate yeast sirtuin protein, $\operatorname{Sir} 2$, a nicotinamide adenine dinucleotide (NAD)-dependent class III histone deacetylase. Besides influencing many other functions, yeast Sir2 extends the animal lifespan $[25,84,85]$. In nematodes and fruit flies, Sir2 also mediates the nutrient-sensing pathway of aging [66, 86]. In rodents and human there are seven Sir2 homologues (SIRT1-7) that have been identified [87-90] and they express ubiquitously across different types of tissues [87, 88, 91]. The recent literature on mammalian SIRT1, the most studied sirtuin protein and generally implicated in longevity, suggests that its expression is highly dependent on the nature of stimuli and the type of tissue [92]. SIRT1 is generally upregulated in diseases/stress states such as, cancer, inflammation, neurodegeneration, DNA damage, oxidative stress, senescence, and CR, perhaps as a protective response [93101], but may be downregulated during aging [92]. Under normal conditions, transcription factor p53 binds to two specific promoter sites of SIRT1 gene and block SIRT1 transcription, under mild stress, forkhead box O3a (FOXO3a) transcription factor associates with p53 and prevents its binding to the promoter site and activates the SIRT1 function such that it deacetylates E2F1, another transcription factor that binds to the SIRT1 promoter site and promotes SIRT1 transcription [92].

There are two isomeric forms of resveratrol: biologically inactive cis-resveratrol and active trans-RES (3,5,4'trihydroxystilbene). Trans-resveratrol (or simply resveratrol) exhibits anti-aging effects by inducing SIRT1 protein in organisms ranging from yeasts to mammals [5, 6, 68, 102]. Although the beneficial effects of resveratrol to the eye are rapidly emerging in the literature, SIRT1 function in the eye has not been widely investigated. Because SIRT1-deficient mice have exhibited defects in the multiple layers of neuronal retina [103], SIRT1 protein possesses crucial but yet to be defined functions in the eye. Interestingly, in $\mathrm{SirT}^{-1-} \mathrm{ES}$, $293 \mathrm{~T}$ cells, SIRT1 did not affect p53-mediated apoptosis
[104], and in Drosophila eye, overexpression of Sir2 promotes caspase-dependent but p53-independent apoptosis that is mediated by the Jun kinase (JNK) and FOXO signaling pathways [105]. In rat undifferentiated E1A-NR3 retinal cells, resveratrol-induced SIRT1 expression increased the activation of $\mathrm{Ku} 70$ and reduced the production of Bax and prevented antibody induced retinal cell death [74]. A polymorphism in complement factor $\mathrm{H}(\mathrm{CFH})$ may increase the risk for ARMD [76]. In human ARPE-19 cells, the overexpression of SIRT1 attenuated FOXO3 recruitment to the $\mathrm{CFH}$ regulatory region and reversed the $\mathrm{H}_{2} \mathrm{O}_{2}$-induced repression of $\mathrm{CFH}$ gene expression [76]. In C33A retinoblastoma $(R b$-null) cells, the overexpression of SIRT1 deacetylated and inactivated $\mathrm{Rb}$ protein, where as inactivation of SIRT1 by siRNA increased the accumulation of $\mathrm{Rb}$ protein [106]. These studies strongly suggest that SIRT1 protein is likely to affect many critical ocular functions that are yet to be substantiated.

\section{Benefits of Sulforaphane in Eye Disease}

Many cruciferous vegetables such as, broccoli sprouts, cabbage, cauliflower, mustard, radish, and turnip contain a class of naturally occurring phytochemicals called isothiocyanates [107]. One such chemical is glucosinolate glucoraphanin, a precursor to sulforaphane [108, 109]. On fungal attack to the plant or injury by chewing, glucosinolate glucoraphanin is converted into active sulforaphane by an enzymatic activity involving myrosinase [107, 110]. Suforaphane is known to provide protection against many types of cancers predominantly by suppressing phase 1 enzymes such as cytochrome P450 and activating phase 2 enzymes [110,111]. Oxidants and electrophiles produced intrinsically or from extrinsic sources function as toxicants and accelerate not only the aging process but also cause numerous age-related pathologies. Phase 2 enzymes produced within the body detoxify these toxicants and protect the organism from injury. Examples of prominent phase 2 enzymes include glutathione transferases (GSTs), glutathione reductase (GSR), glutathione peroxidase (GPX), UDP-glucuronosyltransferases (UGTs), and NAD(P)H:quinone oxidoreductase 1 (NQO1), thioredoxin reductase (TrxR), and heme oxygenase 1 (HO-1) [26]. These phase 2 genes are activated by Keap1/Nrf2/ARE pathway [26]. Under normal conditions, Keap1 binds to the transcription factor $\mathrm{Nrf2}$, retains it in the cytoplasm and promotes its protesomal degradation. However, in the presence of stress-inducers, Keap1 becomes highly reactive and changes its conformation leading to the release of Nrf2. The free Nrf2 moves from cytoplasm to nucleus, where upon interacting with small Maf (sMaf) transcription factor binds to ARE and promote transcription of cytoprotective phase 2 genes [110].

In human ARPE-19 cells, an in vitro model for ARMD, sulforaphane $(0.62-5 \mu \mathrm{M})$ provided protection against the ROS toxicity induced by oxidative stressors (menadione, tert-butyl hydroperoxide, 4-hydroxynonenal, and peroxynitrite) by upregulating phase 2 proteins such as, glutathione (GSH) and quinone reductase (QR) [112]. Similarly, sulforaphane or a bis-2-hydroxybenzylideneacetone (2-HBA) pre-treatments provided protection against retinaldehyde photosensitized oxidation of ARPE cells and fibroblast cell lines from 13.5-day-old embryos of several double-knockout mice, by inducing NQO1 and GSH [113, 114]. In these cells, 
the PI3K/Akt pathway played an important role in regulating Nrf2-dependent activation of ARE and protection against oxidants [115]. Not just sulforaphane, other phytochemicals such as, fisetin, quercetin, myricetin, eriodictyol, taxifolix, epicatechin, epigallocatechin-3-gallate, and zinc metal induced the expression of Nrf2, GSH, NQO1, HO-1, glutamate-cysteine lygase (GCL) [116-118]. In male BALB/c mice, both oral and i.p. administered sulforaphane (0.1 -0.5 $\mathrm{mg}$ ) induced thioredoxin (Trx) in mice retina and protected the retina against light-induced damage and in human K1034 RPE cells, sulforahane induced the binding of Nrf2, small Maf, and c-Jun to the ARE of the Trx gene [119]. Further, similarly in bright-cyclic-light-reared albino SpragueDawley rats, sulforaphane treatment increased upregulation of the retinal levels of Trx, thioredoxin reductase (TrxR), and proteins modified by 4-hydroxynonenal (4-HNE), in addition to increased nuclear translocation of Nrf2 and the DNA binding activity of Nrf2, small Maf, and cJun to the ARE [120]. In the same study, in $661 \mathrm{~W}$ photoreceptor cells, pretreatment with a sub-lethal dose of 4-HNE protected cells against $\mathrm{H}_{2} \mathrm{O}_{2}$-induced cell damage, by upregulating cellular Trx, TrxR, and HO-1 levels in addition to DNA binding activity of Nrf2, small Maf, and cJun to the ARE [120]. Both in Tubby mice \& $\mathrm{C} 57 \mathrm{BL} / 6 \mathrm{~J}$ mice, sulforaphane-induced upregulation of Trx, TrxR, Nrf2 was associated with increased activation of ERKs in preventing photoreceptor degeneration [121]. In addition, in $661 \mathrm{~W}$ photoreceptor cells, sulforaphane and minocycline inhibited light-induced photoreceptor apoptosis partly by downregulating nuclear factorkappaB (NF-кB) p65 subunit, but not effecting mitogenactivated protein kinase (MAPK) [122]. Similarly, in retinal microglial cells derived from Sprague-Dawley rats, these compounds inhibited lipopolysaccharide-induced retinal microglial activation by suppressing the production of inducible nitric oxide synthase (iNOS) and IL-10 [123]. These studies provide compelling evidence on the protective role of Keap1/Nrf2/ARE pathway and its cross-talk with different types of kinases in eye disease.

\section{Benefits of Curcumin in Eye Disease}

Curcumin is the main bioactive compound derived from the rhizomes (thick, modified roots) of the turmeric plant. It has been traditionally used for centuries in Indian cooking and in Ayurvedic medicine for the treatment of most debilitating diseases. In the last couple of decades, curcumin is one of the most intensively studied herbal drugs across all major divisions of medicine. This renewed scientific interest is frequently attributed to its numerous health benefits in several pathologies including eye diseases. The current eye literature seems to suggest that curcumin is most beneficial in the eye as an anti-inflammatory drug.

Curcumin at $0.002 \%$ (but not $0.01 \%$ ) delayed the onset and maturation of galactose-induced cataract in SpragueDawley rats by exerting antioxidant and antiglycating effects, as it inhibited lipid peroxidation, AGE-fluorescence, and protein aggregation [124]. In Wistar NIN rats, curcumin $(0.002-0.01 \%)$ and turmeric $(0.5 \%)$ delayed the progression and maturation of STZ-induced diabetic cataract in a dosedependent manner by reversing the hyperglycemia-induced oxidative stress, with turmeric being more effective than the corresponding dosage level of curcumin [125]. A pretreatment $(24 \mathrm{~h})$ with curcumin $(15 \mu \mathrm{M})$ protected cells against N-methyl-D-aspartic acid (NMDA)-induced excitotoxicity by reducing the intracellular calcium rise in primary retinal cell culture from Wistar rats [126]. Further, in streptotocin (STZ)-induced diabetic Lewis rat retina, curcumin $(0.05 \%)$ restored antioxidant capacity, reduced oxidatively modified DNA (8-OHdG) and nitrotyrosine levels, and provided partial beneficial effects on GSH, in addition to reducing the diabetes-induced elevation of inflammatory factors IL-1 $\beta$, VEGF and NF- $\kappa B$ [127]. In 661W and ARPE-19 cells, in vitro models for AMD, curcumin protected cells from $\mathrm{H}_{2} \mathrm{O}_{2}$-induced cell death and upregulated protective enzymes, such as HO-1, Trx1, and Nrf2 [128]. These studies clearly suggest that in age-related eye diseases, curcumin appears to trigger Keap1/Nrf2/ARE pathway and protect eye by upregulation of cytoprotective phase 2 genes.

Uveitis is the most prominent inflammatory response, occurring in the middle, pigmented layer of the eye called uvea (iris, ciliary body, and choroid) as well as in adjacent tissues including retina. The animals models for uveitis suggest that the process of blood cell leakage is a function of complex, well-orchestrated molecular interactions involving two critical early events: (1) activated blood cellslymphocytes (CD4+ T helper cell subtypes Th1 and Th17 cells), monocytes (macrophages), and granular leukocytes (neutrophils), and (2) inflamed endothelial cells in the blood vessels. Activated Th1 cells secrete inflammatory cytokines such as, interferon-gamma (IFN- $\gamma$ ), interleukin-6 (IL-6), and tumor necrosis factor-alpha (TNF- $\alpha$ ). Besides secreting TNF- $\alpha$ and IL-6, Th17 cells also secrete IL-17, a cytokine that participates in the pathology of uveitis. The secreted cytokines trigger the production of cell adhesion molecules by endothelial and other surrounding cells. In a single published article on the efficacy of curcumin in the management of chronic anterior uveitis, a group of patients who received oral administration of $375 \mathrm{mg}$ of curcumin 3 times a day for 12 weeks, showed efficacy and recurrence rate comparable to corticosteroid therapy but without any adverse drug effects [129]. Topical application of aqueous solution of turmeric $(0.1 \%)$ to rabbit eyes ( 3 times/day for 3 days) prior to the induction of LPS endotoxin-induced uveitis (EIU) significantly reduced the clinical symptoms of EIU [130]. Curcumin $(10-30 \mu \mathrm{M})$ inhibited stroma-derived factor- $1 \alpha$ (SDF$1 \alpha)$-induced CXCR4 cytokine receptor signaling in human retinal endothelial cells (HRECs) and inhibited the growth of new blood vessels in proliferative diabetic retinopathy [131]. In addition, it dose-dependently reduced the viability of HRECs and at $10 \mu \mathrm{M}$ concentration inhibited high glucoseinduced proliferation of HRECs by attenuating VEGFinduced signaling [132]. In a recent study, the inflammatory cytokine TNF- $\alpha$ disrupted the barrier function by causing disappearance of zonula occluden-1 (ZO-1) protein from tight junctions and this disruption was prevented by curcumin $(5-10 \mu \mathrm{M})$ through blocking of NF-kB protein [133]. Curcumin $(20-100 \quad \mu \mathrm{M})$ also dose-dependently downregulated IL-8 and monocyte chemotactic protein 1 (MCP-1) expressions in human retinal pigment epithelial cells following stimulation with glycated human serum albumin, a cell culture model for studying diabetic retinopathy [134]. These studies strongly suggest that curcumin possesses therapeutic, anti-inflammatory functions and modulates numerous inflammatory pathologies in human eye and elsewhere in the body. 


\section{BIOAVAILABILITY AND SAFETY CONCERNS RE- LATED TO CR MIMETICS}

The bioavailability and safety of a given CRM depends on the following three considerations: (1) absorption by human colon, (2) crossing the blood-retinal barrier, and (3) causing minimal adverse drug effects [135]. Although bioavailability of none of the three prominent CRMs (reseveratrol, sulforaphane, curcumin) specifically in the eye is known, based on their studies in other organs (plasma, intestine, brain), they seem to be as readily bioavailable to the eye as readily as in the brain. Some limited bioavailability data on these three CRMs are discussed below.

\section{Resveratrol}

A broad range of resveratrol doses seem to affect beneficial biological functions in the published in vivo bioavailability studies involving rodents and humans [63, 136-142]. The maximum tolerated dose of resveratrol in mice is $4 \mathrm{~g} / \mathrm{day} / \mathrm{kg}$ body weight for 28 days [143]. In our preliminary studies with Lewis and Sprague-Dawley rats, $100 \mathrm{mg} / \mathrm{kg}$ body weight was well tolerated by the animals, as the animals showed no signs of any adverse drug effects. Bioavailability studies in resveratrol have consistently shown that it forms glucuronide and sulfate conjugates within $30 \mathrm{~min}$ of injection, suggesting a very low bioavailability of original aglycone $[137,141,142,144]$. Red wine and cooked peanuts seem to possess larger quantities of resveratrol rather than white wine and raw peanuts [136]. However, experts believe that many of the useful effects of resveratrol come not only from aglycone but also from the modified resveratrol glucuronide and resveratrol-3-sulfate conjugates that circulate in the blood stream in minute quantities.

\section{Sulforaphane}

Relative to resveratrol and curcumin only a limited number of bioavailability studies have been performed for sulforaphane. When rats were injected intravenously with sulforaphane or fed orally, sulforaphane was absorbed rapidly and showed an absolute bioavailability of $82 \%$ in the plasma and showed a dose-dependent pharmacokinetics [145]. A recent study in humans determined the bioavailability of sulforaphane after the consumption of raw and cooked broccoli [146]. Higher levels of sulforaphane conjugates were detected in blood and sulforaphane-derived mercapturic acid in urine when broccoli was consumed raw than cooked. The consumption of raw broccoli resulted in faster absorption as well as higher bioavailability (37\% for raw versus $3.4 \%$ for cooked) of sulforaphane in the blood [146]. Another bioavailability study involving humans also suggested that bioavailability of isothiocyanates was three times greater in raw broccoli than the cooked broccoli [147].

\section{Curcumin}

A combination of curcumin's broad biological functions, its unique ability to be used as a dietary supplement, apparent lack of toxic effects even at extraordinary quantities, and its cheap availability in the market, make it suitable for the development of most essential preventative drugs for the treatment of high-risk patients. Federal Food and Drug Administration listed curcumin as "generally regarded as safe" drug. Although curcumin is a safe drug, it exhibits poor bioavailability after oral consumption. In a Phase I clinical trial of curcumin with 25 patients suffering from high-risk or pre-malignant lesions, the curcumin dosages were gradually increased from $0.5 \mathrm{~g}$ /day to $1,2,4,6,8,12 \mathrm{mg} /$ day [148]. The peak serum concentrations after 1-2 hours of oral consumption of 4,8 , and $6 \mathrm{~g} /$ day were $0.51 \pm 0.11,0.63 \pm 0.06$, and $1.77 \pm 1.87 \mu \mathrm{M}$, respectively [148]. Although there were no toxic effects at $12 \mathrm{~g} /$ day dose, bulkiness of the drug was not relished. Since then numerous bioavailability studies have been conducted and a general story is that curcumin can easily reach distant organs in the body including liver, lungs, heart, and brain and affect biological functions in minute concentrations. Overall there is a limited oral bioavailability. When administered i.p. or i.v., bioavailability slightly increases but not substantially. The major reasons for the low bioavailability include: rapid metabolism, rapid systemic elimination, and poor absorption by the target tissues [149, 150]. Perhaps its poor bioavailability and yellow color appear to be the main limitations for therapeutics and drug development. Yet, curcumin is considered as the lead molecule for dozens of drugs under development. In the academia and pharmaceutical world alike, there seems to be a race towards developing curcumin formulations that enhance its bioavailability in the target tissue. Many curcumin formulations have been suggested: mixing curcumin with adjuvant piperine that blocks curcumin metabolism, or formulations with nanoparticles, liposomes, micelles, and phospholipid complexes [149, 150-152]. An optimized lipid formulation of curcumin, for example, increased its oral bioavailability by 11 -fold in plasma and 4-fold in the brain compared to curcumin alone formulation (Verdure Sciences, Noblesville, Indiana). Two weeks after administration of lipidated form of curcumin in chow at $500 \mathrm{ppm}$ (or $25 \mathrm{mg} / \mathrm{kg}$ body weight), the drug concentration in the mouse brain was $5.79 \pm 1.22 \mu \mathrm{M}$, which is remarkably well above the $1-2 \mu \mathrm{M}$ range of $\mathrm{EC}_{50}$ required for inhibition of iNOS, IL-1 $\beta$, and $\mathrm{PGE}_{2}$ in Alzheimer's animal models for neuroinflammation [153]. Collective evidence on curcumin bioavailability and molecular functions suggests that concentrations ranging from $10 \mathrm{nM}$ to $50 \mu \mathrm{M}$ ranges are suitable for our in vitro studies with human retinal endothelial cells, and wide ranging doses at milligram levels to even a gram (say $10 \mathrm{mg}$ to $1 \mathrm{~g}$ ) can be safely employed for in vivo studies depending on the formulations.

\section{CONCLUSIONS}

The CRM resveratrol appears to function as a pleiotropic compound that triggers multiple beneficial effects including the activation of both anti-aging sirtuin and Keap1/Nrf2/ARE pathways in age-related and eye diseases that occur in all ages. Both sulforaphane and curcumin also exhibit pleiotropic beneficial effects that include the Keap1/Nrf2/ARE pathway, but not the sirtuin pathway. While sulforaphane seem to exert its beneficial functions in age-related diseases, curcumin appears to affect beneficial functions in both age-related and diseases such as uveitis that may occur at any age.

\section{ACKNOWLEDGEMENTS}

This work is supported by funds from NIH T32 CAM Research Training in Neuroscience and Stress, NEI grant 1R21EY018708-01A2, and an unrestricted grant to the Casey Eye Institute from the foundation Research to Prevent Blindness, New York, NY. I thank Drs. Justine Smith and Teri Wadsworth for a critical reading of this manuscript. 


\section{REFERENCES}

[1] Wolf G. Calorie restriction increases life span: a molecular mechanism. Nutr Rev 2006; 64(2 Pt 1): 89-92.

[2] Obin M, Pike A, Halbleib M, Lipman R, Taylor A, Bronson R. Calorie restriction modulates age-dependent changes in the retinas of Brown Norway rats. Mech Ageing Dev 2000; 114(2): 133-47.

[3] Lin SJ, Kaeberlein M, Andalis AA, et al. Calorie restriction extends Saccharomyces cerevisiae lifespan by increasing respiration. Nature 2002; 418(6895): 344-8.

[4] Lin SJ, Ford E, Haigis M, Liszt G, Guarente L. Calorie restriction extends yeast life span by lowering the level of NADH. Genes Dev 2004; 18(1): 12-6.

[5] Sinclair DA. Toward a unified theory of caloric restriction and longevity regulation. Mech Ageing Dev 2005; 126(9): 987-1002.

[6] Bordone L, Guarente L. Calorie restriction, SIRT1 and metabolism: understanding longevity. Nat Rev Mol Cell Biol 2005, 6(4): 298305.

[7] Kyriazis M. Clinical anti-aging hormetic strategies. Rejuvenation Res 2005; 8(2): 96-100.

[8] Calabrese EJ, Baldwin LA. Hormesis: a generalizable and unifying hypothesis. Crit Rev Toxicol 2001; 31(4-5): 353-424.

[9] Radak Z, Chung HY, Goto S. Exercise and hormesis: oxidative stress-related adaptation for successful aging. Biogerontology 2005; 6(1): 71-5.

[10] Johansson L. Hormesis, an update of the present position. Eur J Nucl Med Mol Imaging 2003; 30(6): 921-33.

[11] Cabelof DC, Yanamadala S, Raffoul JJ, Guo Z, Soofi A, Heydari AR. Caloric restriction promotes genomic stability by induction of base excision repair and reversal of its age-related decline. DNA Repair (Amst) 2003; 2(3): 295-307.

[12] Calabrese EJ, Baldwin LA. Tales of two similar hypotheses: the rise and fall of chemical and radiation hormesis. Hum Exp Toxicol 2000; 19(1): 85-97.

[13] Wang Y, Beydoun MA, Liang L, Caballero B, Kumanyika SK. Will all Americans become overweight or obese? Estimating the progression and cost of the US obesity epidemic. Obesity (Silver Spring) 2008; 16(10): 2323-30.

[14] Mattson MP, Cheng A. Neurohormetic phytochemicals: low-dose toxins that induce adaptive neuronal stress responses. Trends Neurosci 2006; 29(11): 632-9.

[15] Mattson MP, Son TG, Camandola S. Viewpoint: mechanisms of action and therapeutic potential of neurohormetic phytochemicals. Dose Response 2007; 5(3): 174-86.

[16] Duncan AM, Phipps WR, Kurzer MS. Phyto-oestrogens. Best Pract Res Clin Endocrinol Metab 2003; 17(2): 253-71.

[17] Nijveldt RJ, van Nood E, van Hoorn DE, Boelens PG, van Norren $\mathrm{K}$, van Leeuwen PA. Flavonoids: a review of probable mechanisms of action and potential applications. Am J Clin Nutr 2001; 74(4): 418-25.

[18] Williams RJ, Spencer JP, Rice-Evans C. Flavonoids: antioxidants or signalling molecules? Free Radic Biol Med 2004; 36(7): 838-49.

[19] Raskin I, Ribnicky DM, Komarnytsky S, et al. Plants and human health in the twenty-first century. Trends Biotechnol 2002; 20(12): 522-31.

[20] Morelli V, Naquin C. Alternative therapies for traditional disease states: menopause. Am Fam Physician 2002; 66(1): 129-34.

[21] Tesch BJ. Herbs commonly used by women: an evidence-based review. Am J Obstet Gynecol 2003; 188(5 Suppl): S44-55.

[22] Ang-Lee MK, Moss J, Yuan CS. Herbal medicines and perioperative care. JAMA 2001; 286(2): 208-16.

[23] Guarente L. Sir2 links chromatin silencing, metabolism, and aging. Genes Dev 2000; 14(9): 1021-6.

[24] Guarente L. SIR2 and aging--the exception that proves the rule. Trends Genet 2001; 17(7): 391-2.

[25] Guarente L. Calorie restriction and SIR2 genes--towards a mechanism. Mech Ageing Dev 2005; 126(9): 923-8.

[26] Kensler TW, Wakabayashi N, Biswal S. Cell survival responses to environmental stresses via the Keap1-Nrf2-ARE pathway. Annu Rev Pharmacol Toxicol 2007; 47: 89-116.

[27] Head KA. Natural therapies for ocular disorders, part one: diseases of the retina. Altern Med Rev 1999; 4(5): 342-59.

[28] Head KA. Natural therapies for ocular disorders, part two: cataracts and glaucoma. Altern Med Rev 2001; 6(2): 141-s66.

[29] Rhone M, Basu A. Phytochemicals and age-related eye diseases. Nutr Rev 2008; 66(8): 465-72.
[30] Congdon N, O'Colmain B, Klaver CC, et al. Causes and prevalence of visual impairment among adults in the United States. Arch Ophthalmol 2004; 122(4): 477-485.

[31] Matthews AG. The lens and cataracts. Vet Clin North Am Equine Pract 2004; 20(2): 393-415, vii.

[32] Robman L, Taylor H. External factors in the development of cataract. Eye 2005 ; 19(10): 1074-82.

[33] Taylor A, Zuliani AM, Hopkins RE, et al. Moderate caloric restriction delays cataract formation in the Emory mouse. FASEB J 1989; 3(6): 1741-6.

[34] Mura CV, Roh S, Smith D, Palmer V, Padhye N, Taylor A. Cataract incidence and analysis of lens crystallins in the water-, ureaand SDS-soluble fractions of Emory mice fed a diet restricted by 40\% in calories. Curr Eye Res 1993; 12(12): 1081-91.

[35] Taylor A, Lipman RD, Jahngen-Hodge J, et al. Dietary calorie restriction in the Emory mouse: effects on lifespan, eye lens cataract prevalence and progression, levels of ascorbate, glutathione, glucose, and glycohemoglobin, tail collagen breaktime, DNA and RNA oxidation, skin integrity, fecundity, and cancer. Mech Ageing Dev 1995; 79(1): 33-57.

[36] Taylor A, Jahngen-Hodge J, Smith DE, et al. Dietary restriction delays cataract and reduces ascorbate levels in Emory mice. Exp Eye Res 1995; 61(1): 55-62.

[37] Gong X, Shang F, Obin M, et al. Antioxidant enzyme activities in lens, liver and kidney of calorie restricted Emory mice. Mech Ageing Dev 1997; 99(3): 181-92.

[38] Li Y, Yan Q, Wolf NS. Long-term caloric restriction delays agerelated decline in proliferation capacity of murine lens epithelial cells in vitro and in vivo. Invest Ophthalmol Vis Sci 1997; 38(1): 100-7.

[39] Li Y, Yan Q, Pendergrass WR, Wolf NS. Response of lens epithelial cells to hydrogen peroxide stress and the protective effect of caloric restriction. Exp Cell Res 1998; 239(2): 254-63.

[40] Wolf NS, Li Y, Pendergrass W, Schmeider C, Turturro A. Normal mouse and rat strains as models for age-related cataract and the effect of caloric restriction on its development. Exp Eye Res 2000; 70(5): 683-92.

[41] Pendergrass WR, Penn PE, Li J, Wolf NS. Age-related telomere shortening occurs in lens epithelium from old rats and is slowed by caloric restriction. Exp Eye Res 2001; 73(2): 221-8.

[42] Wang K, Li D, Sun F. Dietary caloric restriction may delay the development of cataract by attenuating the oxidative stress in the lenses of Brown Norway rats. Exp Eye Res 2004; 78(1): 151-8.

[43] Li D, Sun F, Wang K. Protein profile of aging and its retardation by caloric restriction in neural retina. Biochem Biophys Res Commun 2004; 318(1): 253-8

[44] Reddy GB, Reddy PY, Vijayalakshmi A, Kumar MS, Suryanarayana P, Sesikeran B. Effect of long-term dietary manipulation on the aggregation of rat lens crystallins: role of alpha-crystallin chaperone function. Mol Vis 2002; 8: 298-305.

[45] Chopdar A, Chakravarthy U, Verma D. Age related macular degeneration. BMJ 2003; 326(7387): 485-8.

[46] Katz ML, White HA, Gao CL, Roth GS, Knapka JJ, Ingram DK. Dietary restriction slows age pigment accumulation in the retinal pigment epithelium. Invest Ophthalmol Vis Sci 1993; 34(12): 3297-302.

[47] Sparrow JR, Cai B, Jang YP, Zhou J, Nakanishi K. A2E, a fluorophore of RPE lipofuscin, can destabilize membrane. Adv Exp Med Biol 2006; 572: 63-8.

[48] Sparrow JR, Kim SR, Cuervo AM, Bandhyopadhyayand U. A2E, a pigment of RPE lipofuscin, is generated from the precursor, A2PE by a lysosomal enzyme activity. Adv Exp Med Biol 2008; 613: 393-8.

[49] Gouras P, Ivert L, Landauer N, Mattison JA, Ingram DK, Neuringer M. Drusenoid maculopathy in rhesus monkeys (Macaca mulatta): effects of age and gender. Graefes Arch Clin Exp Ophthalmol 2008; 246(10): 1395-402.

[50] Obin M, Halbleib M, Lipman R, Carroll K, Taylor A, Bronson R. Calorie restriction increases light-dependent photoreceptor cell loss in the neural retina of fischer 344 rats. Neurobiol Aging 2000; 21(5): 639-45.

[51] Li D, Sun F, Wang K. Caloric restriction retards age-related changes in rat retina. Biochem Biophys Res Commun 2003; 309(2): 457-63. 
[52] Mandal AK. Current concepts in the diagnosis and management of developmental glaucomas. Indian J Ophthalmol 1993; 41(2): 5170 .

[53] Foster PJ, Machin D, Wong TY, et al. Determinants of intraocular pressure and its association with glaucomatous optic neuropathy in Chinese Singaporeans: the Tanjong Pagar Study. Invest Ophthalmol Vis Sci 2003; 44(9): 3885-91.

[54] Hille K, Moustafa B, Hille A, Ruprecht KW. Drainage devices in glaucoma surgery. Klin Oczna 2004; 106(4-5): 670-81.

[55] Coleman AL, Miglior S. Risk factors for glaucoma onset and progression. Surv Ophthalmol 2008; 53(Suppl 1): S3-10.

[56] Kawai SI, Vora S, Das S, Gachie E, Becker B, Neufeld AH. Modeling of risk factors for the degeneration of retinal ganglion cells after ischemia/reperfusion in rats: effects of age, caloric restriction, diabetes, pigmentation, and glaucoma. FASEB J 2001; 15(7): 1285-7.

[57] Neufeld AH, Gachie EN. The inherent, age-dependent loss of retinal ganglion cells is related to the lifespan of the species. Neurobiol Aging 2003; 24(1): 167-72.

[58] Li Y, Wolf NS. Effects of age and long-term caloric restriction on the aqueous collecting channel in the mouse eye. J Glaucoma 1997; 6(1): 18-22.

[59] Kim KY, Ju WK, Neufeld AH. Neuronal susceptibility to damage: comparison of the retinas of young, old and old/caloric restricted rats before and after transient ischemia. Neurobiol Aging 2004; 25(4): 491-500.

[60] Shindler KS, Ventura E, Rex TS, Elliott P, Rostami A. SIRT1 activation confers neuroprotection in experimental optic neuritis. Invest Ophthalmol Vis Sci 2007; 48(8): 3602-9.

[61] Nanji AA, French SW. Alcoholic beverages and coronary heart disease. Atherosclerosis 1986; 60(2): 197-8.

[62] Obisesan TO, Hirsch R, Kosoko O, Carlson L, Parrott M. Moderate wine consumption is associated with decreased odds of developing age-related macular degeneration in NHANES-1. J Am Geriatr Soc 1998; 46(1): 1-7.

[63] de la Lastra CA, Villegas I. Resveratrol as an anti-inflammatory and anti-aging agent: mechanisms and clinical implications. Mol Nutr Food Res 2005; 49(5): 405-30.

[64] Fremont L, Belguendouz L, Delpal S. Antioxidant activity of resveratrol and alcohol-free wine polyphenols related to LDL oxidation and polyunsaturated fatty acids. Life Sci 1999; 64(26): 251121.

[65] Pervaiz S. Resveratrol: from grapevines to mammalian biology. FASEB J 2003; 17(14): 1975-85.

[66] Parker JA, Arango M, Abderrahmane S, et al. Resveratrol rescues mutant polyglutamine cytotoxicity in nematode and mammalian neurons. Nat Genet 2005; 37(4): 349-50.

[67] Araki T, Sasaki Y, Milbrandt J. Increased nuclear NAD biosynthesis and SIRT1 activation prevent axonal degeneration. Science 2004; 305(5686): 1010-3.

[68] Anekonda TS. Resveratrol-A boon for treating Alzheimer's disease? Brain Res Brain Res Rev 2006; 52(2): 316-26.

[69] Gao ZB, Hu GY. Trans-resveratrol, a red wine ingredient, inhibits voltage-activated potassium currents in rat hippocampal neurons. Brain Res 2005; 1056(1): 68-75.

[70] Sinha K, Chaudhary G, Gupta YK. Protective effect of resveratrol against oxidative stress in middle cerebral artery occlusion model of stroke in rats. Life Sci 2002; 71(6): 655-65.

[71] Gupta YK, Chaudhary G, Srivastava AK. Protective effect of resveratrol against pentylenetetrazole-induced seizures and its modulation by an adenosinergic system. Pharmacology 2002; 65(3): 1704.

[72] Sparrow JR, Vollmer-Snarr HR, Zhou J, et al. A2E-epoxides damage DNA in retinal pigment epithelial cells. Vitamin E and other antioxidants inhibit A2E-epoxide formation. J Biol Chem 2003; 278(20): 18207-13.

[73] Doganay S, Borazan M, Iraz M, Cigremis Y. The effect of resveratrol in experimental cataract model formed by sodium selenite. Curr Eye Res 2006; 31(2): 147-53.

[74] Anekonda TS, Adamus G. Resveratrol prevents antibody-induced apoptotic death of retinal cells through upregulation of Sirt1 and Ku70. BMC Res Notes 2008; 1: 122.

[75] King RE, Kent KD, Bomser JA. Resveratrol reduces oxidation and proliferation of human retinal pigment epithelial cells via extracellular signal-regulated kinase inhibition. Chem Biol Interact 2005; 151(2): 143-9.
[76] Wu Z, Lauer TW, Sick A, Hackett SF, Campochiaro PA. Oxidative stress modulates complement factor $\mathrm{H}$ expression in retinal pigmented epithelial cells by acetylation of FOXO3. J Biol Chem 2007; 282(31): 22414-25.

[77] Haufschild T, Kaiser HJ, Preisig T, Pruente C, Flammer J. Influence of red wine on visual function and endothelin-1 plasma level in a patient with optic neuritis. Ann Neurol 2003; 53(6): 825-6.

[78] Nagaoka T, Hein TW, Yoshida A, Kuo L. Resveratrol, a component of red wine, elicits dilation of isolated porcine retinal arterioles: role of nitric oxide and potassium channels. Invest Ophthalmol Vis Sci 2007; 48(9): 4232-9.

[79] Liu Q, Ju WK, Crowston JG, et al. Oxidative stress is an early event in hydrostatic pressure induced retinal ganglion cell damage. Invest Ophthalmol Vis Sci 2007; 48(10): 4580-9.

[80] Luna C, Li G, Liton PB, et al. Resveratrol prevents the expression of glaucoma markers induced by chronic oxidative stress in trabecular meshwork cells. Food Chem Toxicol 2009; 47(1): 198-204.

[81] Brakenhielm E, Cao R, Cao Y. Suppression of angiogenesis, tumor growth, and wound healing by resveratrol, a natural compound in red wine and grapes. FASEB J 2001; 15(10): 1798-800.

[82] van Ginkel PR, Darjatmoko SR, Sareen D, et al. Resveratrol inhibits uveal melanoma tumor growth via early mitochondrial dysfunction. Invest Ophthalmol Vis Sci 2008; 49(4): 1299-306.

[83] Sareen D, van Ginkel PR, Takach JC, et al. Mitochondria as the primary target of resveratrol-induced apoptosis in human retinoblastoma cells. Invest Ophthalmol Vis Sci 2006; 47(9): 3708-16.

[84] Lamming DW, Wood JG, Sinclair DA. Small molecules that regulate lifespan: evidence for xenohormesis. Mol Microbiol 2004; 53(4): 1003-9.

[85] Guarente L, Picard F. Calorie restriction--the SIR2 connection. Cell 2005; 120(4): 473-82.

[86] Tissenbaum HA, Guarente L. Increased dosage of a sir-2 gene extends lifespan in Caenorhabditis elegans. Nature 2001; 410(6825): 227-30.

[87] Frye RA. Characterization of five human cDNAs with homology to the yeast SIR2 gene: Sir2-like proteins (sirtuins) metabolize NAD and may have protein ADP-ribosyltransferase activity. Biochem Biophys Res Commun 1999; 260(1): 273-9.

[88] Frye RA. Phylogenetic classification of prokaryotic and eukaryotic Sir2-like proteins. Biochem Biophys Res Commun 2000; 273(2): 793-8.

[89] North BJ, Verdin E. Sirtuins: Sir2-related NAD-dependent protein deacetylases. Genome Biol 2004; 5(5): 224.

[90] North BJ, Schwer B, Ahuja N, Marshall B, Verdin E. Preparation of enzymatically active recombinant class III protein deacetylases. Methods 2005; 36(4): 338-45.

[91] Shi T, Wang F, Stieren E, Tong Q. SIRT3, a mitochondrial sirtuin deacetylase, regulates mitochondrial function and thermogenesis in brown adipocytes. J Biol Chem 2005; 280(14): 13560-7.

[92] Kwon HS, Ott M. The ups and downs of SIRT1. Trends Biochem Sci 2008; 33(11): 517-25.

[93] Gomez-Cabrera MC, Zaragoza R, Pallardo FV, Vina JR. SIRT1 regulation of insulin-signalling pathways in liver, white adipose tissue and pancreas during fasting or calorie restriction. Trends Endocrinol Metab 2007; 18(3): 91-2; author reply 93.

[94] Wang F, Nguyen M, Qin FX, Tong Q. SIRT2 deacetylates FOXO3a in response to oxidative stress and caloric restriction. Aging Cell 2007; 6(4): 505-14.

[95] Hwang JH, Kim DW, Jo EJ, et al. Pharmacological stimulation of NADH oxidation ameliorates obesity and related phenotypes in mice. Diabetes 2009; 58(4): 965-74.

[96] Qin W, Zhao W, Ho L, et al. Regulation of forkhead transcription factor FoxO3a contributes to calorie restriction-induced prevention of Alzheimer's disease-type amyloid neuropathology and spatial memory deterioration. Ann N Y Acad Sci 2008; 1147: 335-47.

[97] Suchankova G, Nelson LE, Gerhart-Hines Z, et al. Concurrent regulation of AMP-activated protein kinase and SIRT1 in mammalian cells. Biochem Biophys Res Commun 2009; 378(4): 836-41.

[98] Liu D, Pitta M, Mattson MP. Preventing NAD(+) depletion protects neurons against excitotoxicity: bioenergetic effects of mild mitochondrial uncoupling and caloric restriction. Ann N Y Acad Sci 2008; $1147:$ 275-82.

[99] Ghosh HS. The anti-aging, metabolism potential of SIRT1. Curr Opin Investig Drugs 2008; 9(10): 1095-102.

[100] Suwa M, Nakano H, Radak Z, Kumagai S. Endurance exercise increases the SIRT1 and peroxisome proliferator-activated receptor 
gamma coactivator-1alpha protein expressions in rat skeletal muscle. Metabolism 2008; 57(7): 986-98.

[101] Chen D, Bruno J, Easlon E, et al. Tissue-specific regulation of SIRT1 by calorie restriction. Genes Dev 2008; 22(13): 1753-7.

[102] Dirks AJ, Leeuwenburgh C. Caloric restriction in humans: potential pitfalls and health concerns. Mech Ageing Dev 2006; 127(1): 1-7.

[103] Cheng HL, Mostoslavsky R, Saito S, et al. Developmental defects and p53 hyperacetylation in Sir2 homolog (SIRT1)-deficient mice. Proc Natl Acad Sci USA 2003; 100(19): 10794-9.

[104] Kamel C, Abrol M, Jardine K, He X, McBurney MW. SirT1 fails to affect p53-mediated biological functions. Aging Cell 2006; 5(1): 81-8.

[105] Griswold AJ, Chang KT, Runko AP, Knight MA, Min KT. Sir2 mediates apoptosis through JNK-dependent pathways in Drosophila. Proc Natl Acad Sci USA 2008; 105(25): 8673-8.

[106] Wong S, Weber JD. Deacetylation of the retinoblastoma tumour suppressor protein by SIRT1. Biochem J 2007; 407(3): 451-60.

[107] Fimognari C, Lenzi M, Hrelia P. Interaction of the isothiocyanate sulforaphane with drug disposition and metabolism: pharmacological and toxicological implications. Curr Drug Metab 2008; 9(7): 668-78.

[108] Zhang Y, Kensler TW, Cho CG, Posner GH, Talalay P. Anticarcinogenic activities of sulforaphane and structurally related synthetic norbornyl isothiocyanates. Proc Natl Acad Sci USA 1994; 91(8): 3147-50.

[109] Zhang Y, Talalay P, Cho CG, Posner GH. A major inducer of anticarcinogenic protective enzymes from broccoli: isolation and elucidation of structure. Proc Natl Acad Sci USA 1992; 89(6): 2399403.

[110] Dinkova-Kostova AT, Talalay P. Direct and indirect antioxidant properties of inducers of cytoprotective proteins. Mol Nutr Food Res 2008; 52 (Suppl 1): S128-38.

[111] Dinkova-Kostova AT, Cheah J, Samouilov A, et al. Phenolic Michael reaction acceptors: combined direct and indirect antioxidant defenses against electrophiles and oxidants. Med Chem 2007; 3(3): 261-8.

[112] Gao X, Dinkova-Kostova AT, Talalay P. Powerful and prolonged protection of human retinal pigment epithelial cells, keratinocytes, and mouse leukemia cells against oxidative damage: the indirect antioxidant effects of sulforaphane. Proc Natl Acad Sci USA 2001; 98(26): 15221-6.

[113] Gao X, Talalay P. Induction of phase 2 genes by sulforaphane protects retinal pigment epithelial cells against photooxidative damage. Proc Natl Acad Sci USA 2004; 101(28): 10446-51.

[114] del VCM, Reyes JM, Park CY, et al. Demonstration by redox fluorometry that sulforaphane protects retinal pigment epithelial cells against oxidative stress. Invest Ophthalmol Vis Sci 2008; 49(6): 2606-12.

[115] Wang L, Chen Y, Sternberg P, Cai J. Essential roles of the PI3 kinase/Akt pathway in regulating Nrf2-dependent antioxidant functions in the RPE. Invest Ophthalmol Vis Sci 2008; 49(4): 1671-8.

[116] Hanneken A, Lin FF, Johnson J, Maher P. Flavonoids protect human retinal pigment epithelial cells from oxidative-stress-induced death. Invest Ophthalmol Vis Sci 2006; 47(7): 3164-77.

[117] Ha KN, Chen Y, Cai J, Sternberg P Jr. Increased glutathione synthesis through an ARE-Nrf2-dependent pathway by zinc in the RPE: implication for protection against oxidative stress. Invest Ophthalmol Vis Sci 2006; 47(6): 2709-15.

[118] Johnson JL, Maher PA, Hanneken AM. The flavonoid, eriodictyol, induces long-term protection in ARPE-19 cells through its effects on Nrf2 activation and phase II gene expression. Invest Ophthalmol Vis Sci 2009; 50(5): 2398-406.

[119] Tanito M, Masutani H, Kim YC, Nishikawa M, Ohira A, Yodoi J. Sulforaphane induces thioredoxin through the antioxidantresponsive element and attenuates retinal light damage in mice. Invest Ophthalmol Vis Sci 2005; 46(3): 979-87.

[120] Tanito M, Agbaga MP, Anderson RE. Upregulation of thioredoxin system via Nrf2-antioxidant responsive element pathway in adaptive-retinal neuroprotection in vivo and in vitro. Free Radic Biol Med 2007; 42(12): 1838-50.

[121] Kong L, Tanito M, Huang Z, et al. Delay of photoreceptor degeneration in tubby mouse by sulforaphane. J Neurochem 2007; 101(4): 1041-52.

[122] Yang LP, Zhu XA, Tso MO. Role of NF-kappaB and MAPKs in light-induced photoreceptor apoptosis. Invest Ophthalmol Vis Sci 2007; 48(10): 4766-76.
[123] Yang LP, Zhu XA, Tso MO. Minocycline and sulforaphane inhibited lipopolysaccharide-mediated retinal microglial activation. Mol Vis 2007; 13: 1083-93.

[124] Suryanarayana P, Krishnaswamy K, Reddy GB. Effect of curcumin on galactose-induced cataractogenesis in rats. Mol Vis 2003; 9: 223-30.

[125] Suryanarayana P, Saraswat M, Mrudula T, Krishna TP, Krishnaswamy K, Reddy GB. Curcumin and turmeric delay streptozotocininduced diabetic cataract in rats. Invest Ophthalmol Vis Sci 2005; 46(6): 2092-9.

[126] Matteucci A, Frank C, Domenici MR, et al. Curcumin treatment protects rat retinal neurons against excitotoxicity: effect on $\mathrm{N}$ methyl-D: -aspartate-induced intracellular $\mathrm{Ca}(2+)$ increase. Exp Brain Res 2005; 167(4): 641-8.

[127] Kowluru RA, Chan PS. Oxidative stress and diabetic retinopathy. Exp Diabetes Res 2007; 2007: 43603.

[128] Mandal MN, Patlolla JM, Zheng L, et al. Curcumin protects retinal cells from light-and oxidant stress-induced cell death. Free Radic Biol Med 2009; 46(5): 672-9.

[129] Lal B, Kapoor AK, Asthana OP, et al. Efficacy of curcumin in the management of chronic anterior uveitis. Phytother Res 1999; 13(4): 318-22.

[130] Gupta SK, Agarwal R, Srivastava S, et al. The anti-inflammatory effects of Curcuma longa and Berberis aristata in endotoxininduced uveitis in rabbits. Invest Ophthalmol Vis Sci 2008; 49(9): 4036-40.

[131] Sameermahmood Z, Balasubramanyam M, Saravanan T, Rema M Curcumin modulates SDF-1alpha/CXCR4-induced migration of human retinal endothelial cells (HRECs). Invest Ophthalmol Vis Sci 2008; 49(8): 3305-11.

[132] Premanand C, Rema M, Sameer MZ, Sujatha M, Balasubramanyam M. Effect of curcumin on proliferation of human retinal endothelial cells under in vitro conditions. Invest Ophthalmol Vis Sci 2006; 47(5): 2179-84.

[133] Kimura K, Teranishi S, Fukuda K, Kawamoto K, Nishida T. Delayed disruption of barrier function in cultured human corneal epithelial cells induced by tumor necrosis factor-alpha in a manner dependent on NF-kappaB. Invest Ophthalmol Vis Sci 2008; 49(2): 565-71.

[134] Bian ZM, Elner VM, Yoshida A, Kunkel SL, Elner SG. Signaling pathways for glycated human serum albumin-induced IL-8 and MCP-1 secretion in human RPE cells. Invest Ophthalmol Vis Sci 2001; 42(7): 1660-8

[135] Anekonda TS, Reddy PH. Can herbs provide a new generation of drugs for treating Alzheimer's disease? Brain Res Brain Res Rev 2005; 50(2): 361-76.

[136] Baur JA, Sinclair DA. Therapeutic potential of resveratrol: the in vivo evidence. Nat Rev Drug Discov 2006; 5(6): 493-506.

[137] Marier JF, Vachon P, Gritsas A, Zhang J, Moreau JP, Ducharme MP. Metabolism and disposition of resveratrol in rats: extent of absorption, glucuronidation, and enterohepatic recirculation evidenced by a linked-rat model. J Pharmacol Exp Ther 2002; 302(1): 369-73.

[138] Vitaglione P, Morisco F, Caporaso N, Fogliano V. Dietary antioxidant compounds and liver health. Crit Rev Food Sci Nutr 2004; 44(7-8): 575-86.

[139] Vitaglione P, Sforza S, Galaverna G, et al. Bioavailability of transresveratrol from red wine in humans. Mol Nutr Food Res 2005; 49(5): 495-504.

[140] Pervaiz S. Chemotherapeutic potential of the chemopreventive phytoalexin resveratrol. Drug Resist Updat 2004; 7(6): 333-44.

[141] Wenzel E, Somoza V. Metabolism and bioavailability of transresveratrol. Mol Nutr Food Res 2005; 49(5): 472-81.

[142] Wenzel E, Soldo T, Erbersdobler H, Somoza V. Bioactivity and metabolism of trans-resveratrol orally administered to Wistar rats. Mol Nutr Food Res 2005; 49(5): 482-94.

[143] Gadducci A, Cosio S, Muraca S, Genazzani AR. Molecular mechanisms of apoptosis and chemosensitivity to platinum and paclitaxe in ovarian cancer: biological data and clinical implications. Eur J Gynaecol Oncol 2002; 23(5): 390-6.

[144] Wang Q, Xu J, Rottinghaus GE, et al. Resveratrol protects against global cerebral ischemic injury in gerbils. Brain Res 2002; 958(2): 439-47.

[145] Hanlon N, Coldham N, Gielbert A, et al. Absolute bioavailability and dose-dependent pharmacokinetic behaviour of dietary doses of 
the chemopreventive isothiocyanate sulforaphane in rat. Br J Nutr 2008; 99(3): 559-64.

[146] Vermeulen M, Klopping-Ketelaars IW, van den Berg R, Vaes WH. Bioavailability and kinetics of sulforaphane in humans after consumption of cooked versus raw broccoli. J Agric Food Chem 2008; 56(22): 10505-9.

[147] Conaway CC, Getahun SM, Liebes LL, et al. Disposition of glucosinolates and sulforaphane in humans after ingestion of steamed and fresh broccoli. Nutr Cancer 2000; 38(2): 168-78.

[148] Cheng AL, Hsu CH, Lin JK, et al. Phase I clinical trial of curcumin, a chemopreventive agent, in patients with high-risk or premalignant lesions. Anticancer Res 2001; 21(4B): 2895-900.

[149] Anand P, Thomas SG, Kunnumakkara AB, et al. Biological activities of curcumin and its analogues (Congeners) made by man and mother nature. Biochem Pharmacol 2008; 76(11): 1590-611.
[150] Anand P, Kunnumakkara AB, Newman RA, Aggarwal BB. Bioavailability of curcumin: problems and promises. Mol Pharm 2007; 4(6): 807-18.

[151] Bisht S, Feldmann G, Soni S, Ravi R, Karikar C, Maitra A. Polymeric nanoparticle-encapsulated curcumin ("nanocurcumin"): a novel strategy for human cancer therapy. J Nanobiotechnol 2007; 5: 3.

[152] Hatcher H, Planalp R, Cho J, Torti FM, Torti SV. Curcumin: from ancient medicine to current clinical trials. Cell Mol Life Sci 2008; 65(11): 1631-52.

[153] Begum AN, Jones MR, Lim GP, et al. Curcumin structurefunction, bioavailability, and efficacy in models of neuroinflammation and Alzheimer's disease. J Pharmacol Exp Ther 2008; 326(1): 196-208.

(C) T.S. Anekonda; Licensee Bentham Open.

This is an open access article licensed under the terms of the Creative Commons Attribution Non-Commercial License (http://creativecommons.org/licenses/by-nc/3.0/) which permits unrestricted, non-commercial use, distribution and reproduction in any medium, provided the work is properly cited. 